\section{MS5-P14 leadXPro - Structure-Based Drug Design on Membrane Protein Targets at SwissFEL}

Robert Cheng, Sandra Markovic, Mathieu Botte, Michael Hennig email: robert.cheng@leadxpro.com

Serial femtosecond crystallography (SFX) using X-ray free electron laser (XFEL) significantly increases the realm of possibility of obtaining structural information from membrane proteins. So far access to the technology is limited to 2 XFEL facilities (LCLS, USA and SACLA, Japan) severely restricting application of this revolutionary technique. LeadXpro brings the industrial expertise of structure-based drug design on membrane protein and has premium access to SwissFEL when it starts operation towards the end of 2017. With capabilities for gene-to-structure, we employ a diversity of technique and industrial expertise including the crystallization of membrane protein (GPCRs, transporters and ion channels), traditional/serial crystallography and electron microscopy, coupled to easy access to synchrotron beamlines at the SLS and the collective scientific expertise at the Paul Scherrer institute. In collaboration with scientists at the SLS, we are also committed to further develop technology for application in serial crystallography. Our goal is to apply these methods for structure-based drug discovery to study difficult membrane protein target implicated in a variety of human diseases. Here we describe the drug discovery pipeline and technology we employed at LeadXPro, and show some examples of the range of activities in the company.

Keywords: XFEL, membrane protein, SwissFEL, LCP, SFX

\section{MS6 Molecular machines and} big complexes

Chairs: Christoph Müller, Miquel Coll

\author{
MS6-P1 Solving the structure of a \\ mammalian acylaminoacyl-peptidase \\ Anna Kiss-Szemán ${ }^{1}$, Veronika Harmat ${ }^{1,2}$, Dóra K. Menyhárd ${ }^{2}$
}

1. Eötvös Loránd University, Institute of Chemistry, Laboratory of Structural Chemistry and Biology adress: Pázmány Péter sétány 1/A, H-1117, Budapest, Hungary tel.:(+36-1) 372-2500/6547

2. MTA-ELTE Protein Modelling Group, adress: Pázmány Péter sétány 1/A, H-1117, Budapest, Hungary tel.:(+36-1) 372-2500/6547, fax: (+36-1) 372-2592 web: prot.chem.elte.hu

email: kszemananna@chem.elte.hu

Acylaminoacyl-peptidases (AAP) catalyse the hydrolysis of oligopeptides, while they function as exopeptidases (processing $\mathrm{N}$-terminal acylated peptides) and endopeptidases too. The mammalian enzyme present also in the human liver - is a key protein in the upstream regulation of the proteasome [1].For members of its enzyme family two basic ways of substrate selection have been discovered so far: flexible domain movement between opened and closed form, or multimerization of rigid monomers. The formation of multimers is linked to the shielding of the "sticky edge" of a $\beta$-sheet - to avoid aggregation. A pernix AAP forms dimers capable for a gating mechanism by domain movements providing size selection of the substrates; in contrast, in $P$. horikoshii AAP formation of hexamers with a complex channel system is responsible for $\beta$-edge shielding and for the size selection too [2]. The goal of our study is to determine how the tetrameric structure of the mammalian AAP is formed: whether to shield the $\beta$-edge, or size selection or both.The mammalian enzyme was purified from porcine (Sus scrofa) liver. We found two crystal forms with their unit cell dimensions being similar: 1) The first reproducible crystals were thin plates diffracting to $5.5 \AA$ A. 2) Using additives a new crystal form was obtained diffracting to $2.8 \AA$, but pseudomerohedrally twinned. The crystallographic phase problem was solved by molecular replacement, using the structure of PhAAP hydrolase domain and a 7-bladed polyalanine propeller domain. Model building and refinement is in progress. Characteristic interactions of the "sticky edge" of a hydrolase domain are formed with the propeller domain of the neighbouring monomer were identified as well as specific interactions responsible for tetramer formation. We propose the role of the insertion found in the third blade of the propeller to be that of shielding the direct entrance to the catalytic triad. This study was supported by BioStruct-X (No.283570) of European Community's 7th Framework Programme; the MedInProt program of the Hungarian Academy of Sciences; OTKA grants NK101072 and PD101095. We acknowledge ESRF and EMBL-Hamburg for providing 